\title{
ON THE IMPACT OF ELECTRONIC REGENERATION ON DYNAMIC WAVELENGTH ROUTING IN SELECTIVELY TRANSPARENT OR OPAQUE WDM NETWORKS
}

\author{
Gerald Franzl and Admela Jukan \\ Vienna University of Technology, Institute of Communication Networks, Favoritenstasse 9/388, \\ A-1040 Vienna, Austria. Admela Jukan currently with Georgia Institute of Technology, School of \\ Electrical and Computer Engineering, Atlanta, GA, USA \\ \{gerald. franzl, admela.jukan\} @tuwien.ac.au
}

\begin{abstract}
We propose a new class of dynamic wavelength routing methods in WDM networks, with a service-differentiated and distributed path quality information, applicable for transparent and selectively opaque network structures, and study ring and mesh WDM networks with long-reach interconnections and short-reach transparent metro-islands.
\end{abstract}

Key words: Electronic regeneration, wavelength routing, QoS, distributed provisioning.

\section{INTRODUCTION}

Fundamental advances in WDM optical technologies (e.g. availability of ultra long-reach transport and all-optical switching) have enabled the deployment of all-optical networks. New high bit-rate network applications are creating the need for transmission channels with service-differentiated offerings. Still most architectures are service type related and not designed to accommodate different service classes which require dedicated and servicetype related transmission quality guarantees. Accurate engineering of analogue WDM spans, is critical if combined with dynamic wavelength routing, as path lengths vary. Optical network architectures that include electronic regeneration at each switching node (opaque optical networks) 
simplify the WDM span design and resolves wavelength continuity constraints. On the other hand electronic regeneration also adds delay, can significantly reduce the connection (and network) reliability and increase operational costs. For optical transport network architectures scalable to application of different service types, the availability of efficient protocols will be critical to enable dynamic, service-differentiated lightpath provisioning.

Here, we propose a generic approach to on-demand provisioning in fully or partially electronically regenerative, constraint-based routed, multiservice WDM networks, suitable for distributed implementation, and applicable to optical network services now emerging. More precisely, we focus on distributed control for dynamic wavelength routing and the performance impact of electronic regeneration in different network topologies. In particular, the usage of electronic regeneration has been shown difficult to resolve with standard graph-theoretical methods due to the negative weights, which are avoided by our approach [1,2]. We have simulated ring and mesh WDM networks with long-reach interconnections and short-reach transparent metro-islands, for which four different link types showing different transmission quality in terms of SNR degradation, reliability and delay are assumed. The results show the capability of our methods to accommodate arbitrary number and type of path properties, related to different network elements for the purpose of transmission quality evaluation as required by new emerging services.

\section{THE NETWORK MODEL}

The network model relies on a distributed definition of the network structure by autonomous groups of optical network elements (NE) and service-specific characterisation of these optical network elements. NE, a term which stands for any manageable physical resource or a group of physical resources along wavelength-routed paths (e.g. fibre connections, fibre amplifiers, wavelength splitters, all-optical nodes or electronic regenerators) that affects the wavelength channel performance.

Based on $[2,3]$, we model the WDM network $G(N, L, \Lambda, S)$ with the number of nodes $N$, WDM links $L$, a pool of wavelengths $\Lambda$, and an optical network service set $S$. The local network state information defined as a vector of values $a_{1}, a_{2}, \ldots$ is related to transmission quality, reliability, manageability and traffic conditions, based on which a choice between a number of feasible paths is made at the destination, according to servicelevel agreements and optical network operational objectives. Given any source-destination pair of nodes within the graph $G$, and the constraints 
$d\left(P_{s d}\right)=\left[d_{1}, d_{2}, \ldots\right]$ on the vector components $a_{1}, a_{2}, \ldots$, correspondingly, the multi-constrained routing problem is to find some feasible paths between a given source-destination $(s d)$ pair of nodes which satisfies bounded requirements put on the path for each component of $\bar{a}\left(\mathrm{P}_{\mathrm{sd}}\right)$. According to the properties of each traversed NE, the operator of the path quality evaluation procedure can be additive (sum), multiplicative (product) or restrictive (minimum value) [3].

To overcome the fundamental complexity of the multi-constrained path selection, we propose a protocol for on-demand wavelength channel provisioning, suitable for distributed implementation in both opaque and alloptical networks, with a local, instead of global, network state update [2]. For illustration, we will here focus on two types of network states: additive (SNR degradation, delay) and multiplicative (reliability) [2]. In addition, we will consider such network architecture where only electronic regenerators, if available, are capable of wavelength shifting, but the presented methods can simply handle any other architecture, e.g. all-optical network deploying all-optical regenerators or all-optical wavelength converters.

\subsection{Distributed provision of selectively regenerative wavelength paths}

Step 1: Get a connection request between ( $s r c$, dest) for a service $S_{r}$, with the service-specific vector of routing constraints on paths, i.e. max. transmission degradation $q_{\max }\left(\mathrm{S}_{\mathrm{r}}\right)$, max. delay $d_{\max }\left(\mathrm{S}_{\mathrm{r}}\right)$, min. reliability $r_{\min }\left(\mathrm{S}_{\mathrm{r}}\right)$. (Note that for a given connection request another type of service would impose other constraints.) Forward this request and the message containing the initial values of $\bar{a}\left[a_{\mathrm{q}}, a_{\mathrm{d}}, a_{\mathrm{r}}\right]$ from $s r c$ to all neighbouring NEs (e.g. $\left[a_{\mathrm{q}}=0, a_{\mathrm{d}}=0, a_{\mathrm{r}}=1\right]$ ) and the corresponding path sequence $p$. In addition to $a, s r c$ and dest, the path information contain the unique path identifier (id), information regarding the wavelength to be allocated and unique identifiers of the visited network elements (path sequence $p$ ).

Step 2: Forward the received path information message with updated values to all neighbours, excluding those which are contained in the path sequence ("visited"), until dest is reached, according to the following rule.

Path information update: For each idle wavelength, all visited $\mathrm{NE}_{\mathrm{k}}$ except electronic regenerators, updates the values of the path information $\bar{a}\left[a_{\mathrm{q}}, a_{\mathrm{d}}, a_{\mathrm{r}}\right]$, so that $a_{\mathrm{q}}$ and $a_{\mathrm{d}}$ are summed along the precedent NEs, while $a_{\mathrm{r}}$ is multiplied. If an electronic regenerator is visited, the parameter $a_{\mathrm{q}}$ is reset to the initial value, while the delay is kept summed, and $a_{\mathrm{r}}$ multiplied.(Wavelength shifting NE) Path information messages which are characterised by wavelength occupancy or by unacceptable network states with respect to $\bar{d}\left(\mathrm{~S}_{\mathrm{r}}\right)$ are discarded and do not reach the destination. 
(Wavelength continuous $N E$ ) Path information messages which are characterised by wavelength discontinuity, occupancy or by unacceptable network states with respect to $\bar{d}\left(\mathrm{~S}_{\mathrm{r}}\right)$ are discarded and do not reach the destination.

If $\mathrm{NE}_{k}$ is not dest, the visited network element $i d$ and the wavelength for allocation are added to the path information of surviving messages, which are forwarded to the neighbouring nodes which will then perform Step 2.

Step 3: From the obtained set of all feasible paths $\mathrm{P}=\left\{p_{\mathrm{id} 1}, p_{\mathrm{id} 2}, ..\right\}$ for which $a_{\mathrm{q}}<q_{\max }\left(\mathrm{S}_{\mathrm{r}}\right), a_{d}<d_{\max }\left(\mathrm{S}_{\mathrm{r}}\right), a_{r}>r_{\min }\left(\mathrm{S}_{\mathrm{r}}\right)$, dest selects the best path according to one of the following criteria: $\mathrm{min}$. hops, $\mathrm{min}$. cost, min. number of traversed NEs of particular type, e.g. wavelength converters, etc. The information available to dest contains path $i d$, all traversed network elements and allocated wavelengths, as well as the path performance expressed in form of the last update of path information $a\left(P_{\mathrm{sd}}\right)$.

Step 4: From dest, send the acknowledgement message back along the selected path to $s r c$, for the purpose of resource reservation. The connection can then be established.



(a)

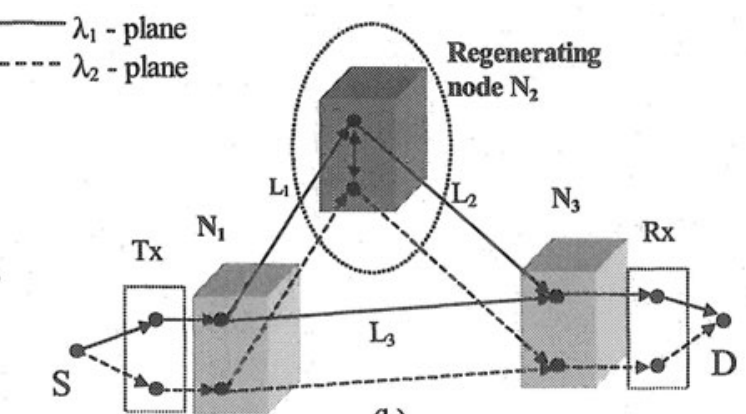

(b)

\begin{tabular}{|c|c|c|c|}
\hline \multicolumn{2}{|c|}{$\mathrm{S}_{1}$-specific NE } & $q[\mathrm{~dB}]$ & $d$ [time units] \\
\hline $\mathrm{Tx} @ \mathrm{~N}_{1}$ & $\lambda_{1}, \lambda_{2}$ & 4 & 1 \\
\hline $\mathrm{Rx} @ \mathrm{~N}_{3}$ & $\lambda_{1}, \lambda_{2}$ & 5 & 1 \\
\hline Reg@ $\mathrm{N}_{2}$ & $\lambda_{1}, \lambda_{2}$ & not applicable & 10 \\
\hline $\mathrm{N}_{1}, \mathrm{~N}_{2}, \mathrm{~N}_{3}$ & $\lambda_{1}, \lambda_{2}$ & 3 & 1 \\
\hline \multirow[t]{2}{*}{$\mathrm{L}_{1}, \mathrm{~L}_{2}$} & $\lambda_{1}$ & 10 & 1 \\
\hline & $\lambda_{2}$ & 12 & 1 \\
\hline \multirow[t]{2}{*}{$\mathrm{L}_{3}$} & $\lambda_{1}$ & \multicolumn{2}{|c|}{$\begin{array}{c}\notin \Lambda_{\text {sws: }} \text { : not avail. for } S_{1} \\
\text { (e.g. reserved or insufficient quality) }\end{array}$} \\
\hline & $\lambda_{2}$ & 6 & 1 \\
\hline
\end{tabular}

(c)

Figure 1.Example network: (a) physical topology, (b) auxiliary graph, (c) S1-specific network state representation.

For illustration, consider an example WDM network with 3 nodes, 3 links and 2 wavelengths per link, capable of accommodating 2 service classes, $S_{1}$ and $S_{2}$ (Fig. 1). Assume that $S_{1}$ is required between $N_{1}$ and $N_{3}$, for 
which the max. degradation of SNR should not exceed $30 \mathrm{~dB}$, i.e. $a_{q}\left(\mathrm{~S}_{1}\right)=30$. For this service type, we consider the following manageable, $S_{1}$-specific NEs: $\operatorname{Tx}\left(\mathrm{N}_{1}\right), \operatorname{Reg}\left(\mathrm{N}_{2}\right), \operatorname{Rx}\left(\mathrm{N}_{3}\right)$, and nodes and links, with their associated service-specific wavelength sets and quality properties. Here, only $\mathrm{N}_{2}$ can provide wavelength conversion for $S_{1}$, due to the $S_{1}$-specific regenerators. The network state information is expressed as a pair value $\left[\begin{array}{ll}a_{q}, & a_{d}\end{array}\right]$, corresponding to transmission quality degradation and delay. Note that the restrictive attributes are automatically taken into account by the definition of $\Lambda_{\text {Sws }}$ : the wavelengths outside $\Lambda_{\text {Sws }}$ are simply excluded from the path discovery (e.g. $L_{3}$ ).

According to the defined wavelength-path provisioning scheme, source $S$ forwards the connection request to its first neighbouring network element (here: Tx), by generating two (being number of wavelengths) pieces of path information of type [path id, dest, actual path information, \{list of visited NEs at (wavelength) $\}]$, i.e. $\left[m k, \mathrm{D}\left(\mathrm{N}_{3}\right),(0,0),\left\{\mathrm{S}\left(\lambda_{1}\right)\right\}\right]$ and $\left[a v,(0,0),\left\{\mathrm{S}\left(\lambda_{2}\right)\right\}\right]$. Transmitter Tx forwards the updated messages $\left[m k, \mathrm{D}\left(\mathrm{N}_{3}\right),(4,1),\left\{\mathrm{S}, \mathrm{Tx}\left(\lambda_{1}\right)\right\}\right]$ and $\left[a v, \mathrm{D}\left(\mathrm{N}_{3}\right),(4,1),\left\{\mathrm{S}, \operatorname{Tx}\left(\lambda_{2}\right)\right\}\right]$ to its neighbour $\mathrm{N}_{1}$, which then has to generate four outgoing messages to its neighbours $\mathrm{N}_{2}$ and $\mathrm{N}_{3}$. Node $\mathrm{N}_{2}$, for example, receives the following messages: $\left[m k, \mathrm{D}\left(\mathrm{N}_{3}\right),(17,3),\left\{\mathrm{S}, \mathrm{Tx}, \mathrm{N}_{1}, \mathrm{~L}_{1}\left(\lambda_{1}\right)\right\}\right]$ and $[a v$, $\left.\mathrm{D}\left(\mathrm{N}_{3}\right),(19,3),\left\{\mathrm{S}, \mathrm{Tx}, \mathrm{N}_{1}, \mathrm{~L}_{1}\left(\lambda_{2}\right)\right\}\right]$. After the regeneration however, since wavelength shifting is possible four messages are sent from $\mathrm{N}_{2}$ to $\mathrm{N}_{3}$, i.e. [mk, $\left.\mathrm{D}\left(\mathrm{N}_{3}\right),(0,14),\left\{\mathrm{S}, \mathrm{Tx}, \mathrm{N}_{1}, \mathrm{~L} 1, \mathrm{~N}_{2}, \operatorname{Reg}\left(\lambda_{1}\right)\right\}\right],\left[a v, \mathrm{D}\left(\mathrm{N}_{3}\right),(0,14),\left\{\mathrm{S}, \mathrm{Tx}, \mathrm{N}_{1}, \mathrm{~L}_{1}, \mathrm{~N}_{2}\right.\right.$, $\left.\left.\operatorname{Reg}\left(\lambda_{2}\right)\right\}\right]$, and two newly generated, wavelength shifted $\left[m i, \mathrm{D}\left(\mathrm{N}_{3}\right),(0,14),\{\mathrm{S}, \mathrm{Tx}\right.$, $\left.\left.\mathrm{N}_{1}, \mathrm{~L}_{1}, \mathrm{~N}_{2}\left(\lambda_{1}\right), \operatorname{Reg}\left(\lambda_{2}\right)\right\}\right],\left[s b, \mathrm{D}\left(\mathrm{N}_{3}\right),(0,14),\left\{\mathrm{S}, \mathrm{Tx}, \mathrm{N}_{1}, \mathrm{~L}_{1}, \mathrm{~N}_{2}\left(\lambda_{2}\right), \operatorname{Reg}\left(\lambda_{1}\right)\right\}\right]$. In this example, the destination may chose the best path in terms of transmission quality, i.e. $\mathrm{Tx}-\mathrm{N}_{1}-\mathrm{L}_{1}-\mathrm{N}_{2}-\operatorname{Reg}\left(\mathrm{N}_{2}\right)-\mathrm{L}_{2}-\mathrm{N}_{3}-\mathrm{Rx}$ at $\lambda_{1}$ (wavelength continuous), with overall transmission degradation $\Sigma q=10+3+5=18(<30)$. Note that the degradation Tx- $\mathrm{N}_{1}-\mathrm{L}_{1}-\mathrm{N}_{2}$ is compensated by $\operatorname{Reg}\left(\mathrm{N}_{2}\right)$ and does not contribute to the overall path degradation. However, if the request on delay for $S_{1}$ was $d\left(\mathrm{~S}_{1}\right) \leq 15$ (time units), this path would show unsatisfactory performance ( $\Sigma d=17$, "regenerator-bottleneck"). On the other hand, without regeneration over the same route, an unacceptable degradation, i.e. $\Sigma q=38$, would be obtained. Another path, e.g. Tx- $\mathrm{N}_{1}-\mathrm{L}_{3}-\mathrm{N}_{3}-\mathrm{Rx}$ at $\lambda_{2}$, however, would satisfy both bounds ( $q$ and $d$ ), i.e. $\Sigma q=21(>18$ but still $<30)$ and $\Sigma d=5<15$. Note that the NE quality attributes and the routing decision completely change, if a service of type $S_{2}$ must be set-up.

\section{PERFORMANCE STUDY}

For simulation results, we adopt the model with optical network services and dynamic wavelength routing as in $[1,2,3,4]$, where the connection 
requests arrive according to Poisson process, call holding time being neg. exp. distributed; traffic distribution and service requests are uniform; confidence level is $95 \%$. We will show here results for ring and mesh-torus network topologies as well as for a larger symmetric network where six ring networks are interconnected (denoted as 6x5 structure). Being less connected, ring networks need fewer messages and show particular performance regarding wavelength shifting; mesh-torus networks allows us to better evaluate scalability and impact of wavelength shifting and/or electronic regeneration and can be viewed as a model case with respect to the average number of hops required in a mesh network [1,4].

In order to analyse networks with differentiated transmission impairments and service requirements, we assume that our networks are designed with four different link types. The links differ in their connection spans, e.g. show different fibre-lengths and different numbers of amplifiers. Tab. 1 shows the parameters of relevance for routing.. We assumed that the parameters of the transmission fibre, described as "transmission degradation", a parameter comprising all relevant transmission attributes, are defined per wavelength, while reliability and delay are assumed to be the same for all wavelengths; long haul links are taken less reliable and show more delay (e.g. Type D).

\begin{tabular}{lllllll}
\hline $\begin{array}{l}\text { Link Type / NE } \\
\mathrm{a}_{\mathrm{a}}[\mathrm{dB}]\end{array}$ & $A$ & $B$ & $C$ & $D$ & Node & Reg. \\
\hline$\lambda_{1}$ & 5 & 12 & 18 & 24 & 3 & $\mathrm{n} / \mathrm{a}$ \\
$\lambda_{2}$ & 4.5 & 10.6 & 15.7 & 20.8 & 3 & $\mathrm{n} / \mathrm{a}$ \\
$\lambda_{3}$ & 3.9 & 9 & 13.4 & 17.6 & 3 & $\mathrm{n} / \mathrm{a}$ \\
$\lambda_{4}, \lambda_{5}$ & 3.4 & 7.7 & 11 & 14.4 & 3 & $\mathrm{n} / \mathrm{a}$ \\
$\lambda_{6}$ & 5 & 12 & 18 & 24 & 3 & $\mathrm{n} / \mathrm{a}$ \\
$\lambda_{7}$ & 6.6 & 16.3 & 25 & 33.6 & 3 & $\mathrm{n} / \mathrm{a}$ \\
$\lambda_{8}$ & 8.2 & 20.6 & 32 & 43.2 & 3 & $\mathrm{n} / \mathrm{a}$ \\
$\mathrm{a}_{\mathrm{r}}[\%]$, all & 99.9 & 98.75 & 97.5 & 96.5 & 99.99 & 99.9 \\
$\mathrm{a}_{\mathrm{d}}[\mathrm{tu}]$, all & 2 & 5.5 & 8.8 & 12 & 0.0 & 10 \\
\hline
\end{tabular}

Tab. 1. Quality attributes per wavelength along different link types.

( $a_{q}$ transmission degradation, $a_{r}$ reliability, $a_{d}$ delay)

As previously stated, all studied networks, in order to achieve different properties per path, deploy the four different WDM link types (Type A, B, C and $D$ ), as defined in Tab. 1. The distribution of these link types in the studied network topologies can be seen in Fig. 2 and Fig. 3.

For mesh-torus and ring networks, we assumed the "islands" of metropolitan networks (shaded), all connected with short links of Type A 
inside the metro-area, while interconnected with the Type $B, C$ or $D$ with long reach fibres (Fig. 2).
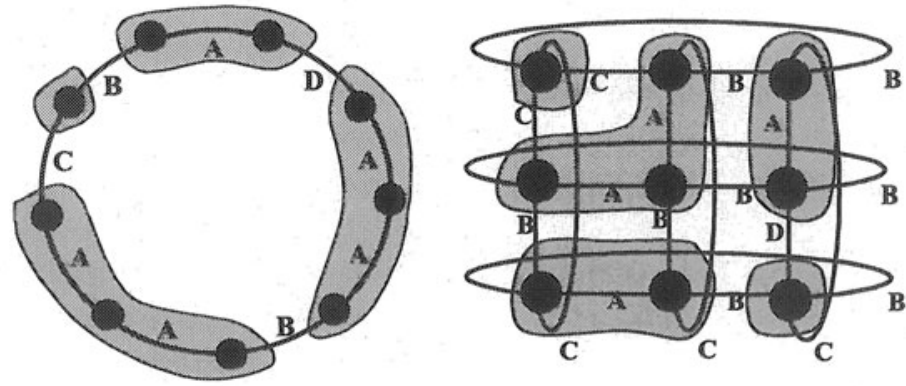

Figure 2. Link type distribution within the studied ring and mesh-torus network.

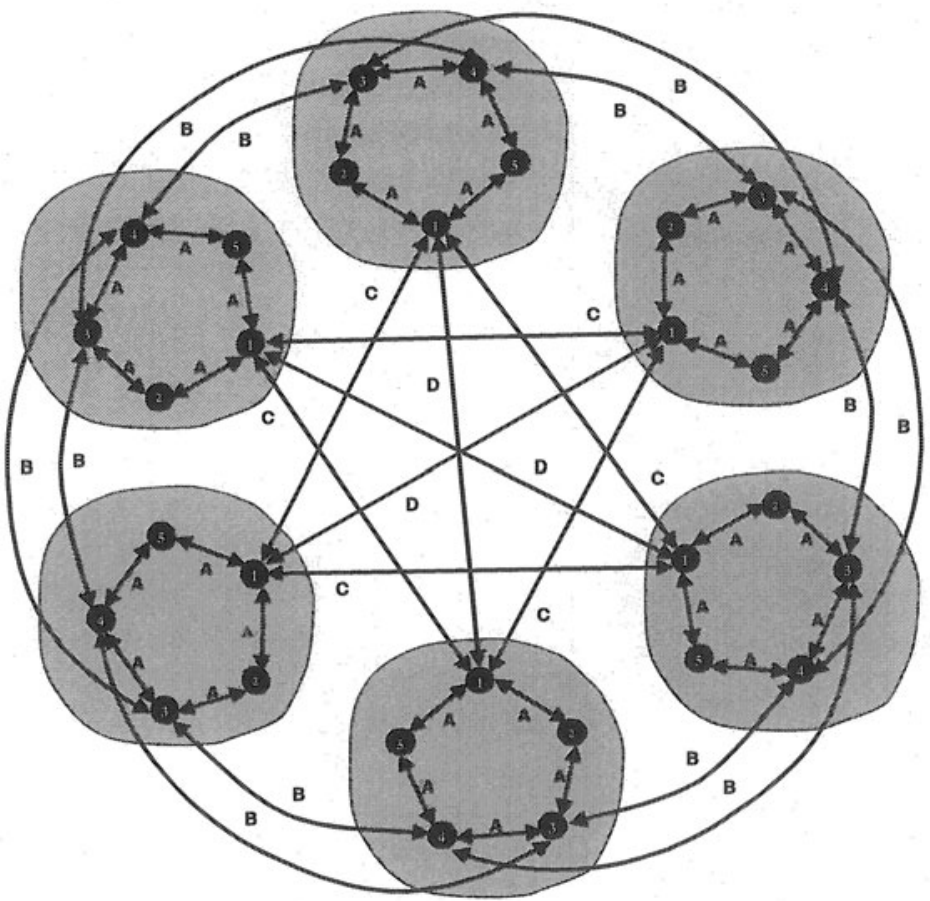

Figure 3. $6 \times 5$ structure and link type distribution within this network.

For the $6 \times 5$ network we assumed the 5-node rings to be the metropolitan networks using links of Type A only, the doubled ring connection of all these metropolitan networks is established with links of Type B while the long haul connections intercepting this city-ring are links of Type $\mathrm{C}$ for the 
connections to the next neighbours; Type D is used to connect 5-node rings diagonally between each other (Fig. 3).

We studied the impact of electronic regeneration on blocking probability by assuming that two effects of regeneration may improve the probability of a successful connection set-up, namely wavelength shifting capability and improvement of transmission quality. We studied all-optical (no el. regeneration), selectively regenerative (sparse el. regeneration along the network) and opaque networks (el. regeneration at each optical node).

For sparse regenerative networks (selectively opaque), the optical nodes equipped with electronic regenerators need to be located as the placement has strong impact on the results. In the ring and mesh-torus topologies the nodes with regeneration and wavelength-shifting capabilities are located at (a) 3 nodes, diagonally for mesh-torus and every third node for ring, and (b) 5 nodes, cross-diagonally for mesh-torus and every second node for ring. In the $6 \times 5$ structure the nodes with regeneration are located (a) at the dedicated nodes of the 5-node rings which home all long distance connections, (b) the nodes of the 5-node rings which home the interconnections to all neighbour 5-node rings, (c) all the nodes of (a) and (b). Additionally we also consider two cases, where the 5-node rings are either totally transparent or opaque: (d) two opposite rings are opaque and (e) every second ring is opaque.

We assumed for simplicity that if regeneration is available, it is available for both studied services. This is generally not the case, since electronic regeneration is likely to be implemented in a service-differentiated manner (e.g. STM-64 line cards within optical nodes). To support multi-service regimes every node in some way needs to handle all services ("block, regenerate or bypass"). If nodes block services, this changes the network structure presented to calls of these services. Additionally, we limited wavelength conversion to one shift per light-path to reduce the complexity of routing by suppressing frequent messaging. Therefore a light-path never uses more than two different wavelengths, which might affect the routing success for the call under consideration, but keeps wavelength-continuos paths for future calls idle. The location of the shift is not restricted, except that only opaque nodes can shift and that source and destination nodes are not allowed to shift the wavelength. The two wavelengths used can be any pair of available wavelengths from the considered wavelength-sets, no additional restriction for wavelength selection is included.

\section{NUMERICAL RESULTS}

In our simulation studies, we assume two different service classes with service requirements given in Fig. 4 to Fig. 6 . We assume that a real-time 
service $\left(S_{1}\right)$ requires less reliability $(60 \%)$, but accepts nearly no transmission delay (20tu), and a data service $\left(S_{2}\right)$ requiring higher reliability $(90 \%)$ while accepting large delays (100tu). The transmission degradation requirement $(40 \mathrm{~dB})$ is identical for both service classes, assuring proper detection at the destination node independent of transmitted service.

While in case of fully transparent networks the blocking probability for $S_{1}$ and $S_{2}$ are identical, the improvement caused by regeneration differs (Fig. 4). In the mesh-torus network for both services the usage of regeneration decreases blocking. For ring networks the usage of regeneration also resolves blocking of initial calls. However, rings have only one alternative path, and if it is allocated, a significant amount of resources becomes busy, which blocks the future calls. This is highly reflected by the delay-sensitive service $S_{1}$. In addition, rings do not benefit of wavelength shifting capability due to the high degree of load correlation $[1,4]$. For the fully opaque ring we find that for $S_{1}$ the delay caused by regeneration restricts the maximum path length to less than the diameter, causing a blocking bias which is present also for very light network loads. The mesh-torus network, on the other hand does not show this bias in blocking probability, as here the diameter of the network is smaller than the maximum span feasible for $S_{1}$.

The 6x5 network topology shows comparable results (Fig. 5). Again, the blocking probability in case of full transparency is identical for $S_{1}$ and $S_{2}$ and improvement by implementation of regeneration capabilities is different. We find biased blocking for $S_{1}$ not only for the fully opaque network but also for the selectively opaque structure (c), where three of the five city-ring nodes are defined as opaque. At lower loads, $S_{1}$ is better served by the networking structures (d) and (e), where dedicated 5-node rings are defined to be opaque, while all other are fully transparent. On the other hand, these structures do not operate $S_{2}$ with efficient quality; structure (b) shows far less blocking probability at a comparable number of opaque nodes. 



Figure 4. Blocking probability per service for ring (o) and mesh-torus (x) network architecture; fully transparent: solid, selectively opaque: dashed (a), dash-dot (b), fully opaque: dotted. 



Figure 5. Blocking probability per service for $6 \times 5$ network architecture; fully transparent: solid, selectively opaque: long dashed (a), short dashed (b), small dotted (c), dash-dot (d),dash-dot-dot (e), fully opaque: big dotted. 


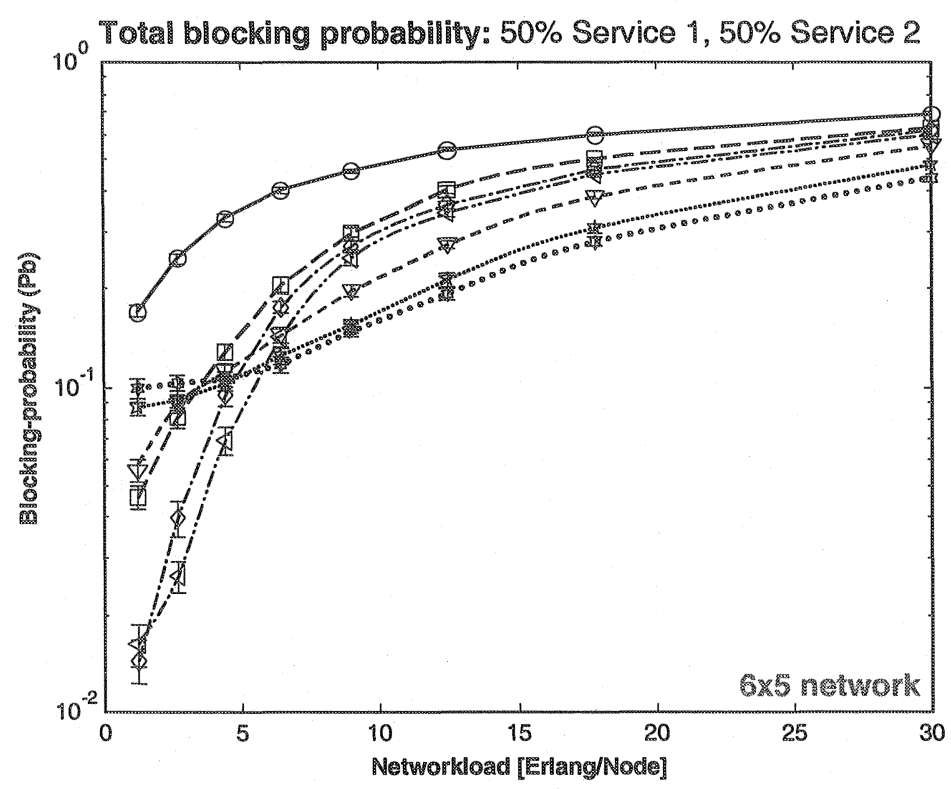

Figure 6. Total blocking probability for $6 \times 5$ network architecture; fully transparent: solid, selectively opaque: long dashed (a), short dashed (b), small dotted (c), dash-dot (d), dash-dot-m dot (e), fully opaque: big dotted.

In a multi-service network, the issues of performance optimisation under multiple constraints are even more complicated. For a multi-service network scenario, it is hard to determine the best network operating point for different types of traffic. On one hand, service-specific traffic should not be affected due to the resource reservation, while on the other hand, the overall network operation and performance should not degrade. To find an optimum operational point for all services the total blocking probability becomes an important issue. In Fig. 6 the overall network blocking probability for the $6 \times 5$ network is shown, with the two service classes $S_{1}$ and $S_{2}$ being uniformly distributed for arrivals.

Depending on the targeted network load or the acceptable blocking probability, a structure which shows a trade-off between these two parameters can be selected. However, we believe that the servicedifferentiated blocking probability will be of primary importance for network providers to analyse. A blocking probability bias as it occurred in the simulation examples, caused by the very tight delay restriction of the real-time service, should be avoided as it indicates that not all sourcedestination combinations are feasible. 


\section{CONCLUSION}

By further studying these and related results for applicability of our method, we have revealed the following issues of constraint-based path selection: (i) distributed protocols are crucial for selectively regenerative and opaque network architecture, since they avoid problems of path algebra to deal with negative weights, if service-differentiated transmission properties characterise a WDM network; however, they do not scale a further improvement for their practicability has to be achieved, (ii) electronic regeneration need a particular consideration for wavelength routing, since it belongs to the class of network elements which at the same time may deteriorate (e.g. delay) and improve (e.g. SNR) performance, and, (iii) whether a network under dynamic traffic regime will profit out of electronic regeneration depends on its topology, operational load, wavelength routing strategy and the guaranteed service levels.

\section{REFERENCES}

[1] M. Kovacevic, et.al: "Electronic wavelength translation in optical networks", IEEE JLT, 1996, pp. 1161-1170.

[2] A. Jukan, et al.: "Distributed provisioning of wavelength channels in multi-service WDM networks", OFC'01, pp. MH3-1, MH3-3.

[3] A. Jukan, et al: "Service-specific resource allocation in WDM networks with quality constraints", IEEE JSAC, 2000, pp. 2051-2061.

[4] S. Subramaniam, et. al:"Connectivity and Sparse Wavelength Conv. in WavelengthRouting Networks", INFOCOM 96, pp.148-155 